\title{
Carbon ion beam radioresistant rodent cells are sensitized to trifluorothymidine exposure
}

\author{
SUNG-JAE BAEK ${ }^{1,2 *}$, KATSUTOSHI SATO $^{3 *}$, NAOHIRO NISHIDA $^{2,4 *}$, JUN KOSEKI $^{5}$, \\ KAZUHIKO HAYASHI $^{1,2}$, KOICHI KAWAMOTO ${ }^{2,4}$, MASAMITSU KONNO ${ }^{2}$, \\ YUICHIRO DOKI $^{4}$, MASAKI MORI ${ }^{4}$, KAZUHIKO OGAWA $^{1}$ and HIDESHI ISHII ${ }^{5}$
}

\author{
Departments of ${ }^{1}$ Radiation Oncology, and ${ }^{2}$ Frontier Science for Cancer and Chemotherapy, Osaka University \\ Graduate School of Medicine, Osaka 565-0871; ${ }^{3}$ National Institute for Radiological Science, Chiba 263-8555; \\ Departments of ${ }^{4}$ Gastroenterological Surgery and ${ }^{5}$ Cancer Profiling Discovery, Osaka University \\ Graduate School of Medicine, Osaka 565-0871, Japan
}

Received March 7, 2016; Accepted February 27, 2018

DOI: $10.3892 / \mathrm{ol} .2018 .9004$

\begin{abstract}
Although charged particle therapy, including carbon ion beam radiation, is a cutting-edge technology in human cancer treatment, the molecular mechanisms underlying cellular resistance to this type of therapy remain unknown. Furthermore, the chemotherapeutic agents that are most effective at overcoming cellular resistance remain unknown. In the present study, carbon ion beam radioresistant rodent cells were developed and their sensitization to trifluorothymidine (FTD), a derivative of deoxythymidine, was studied. The results of the present study demonstrated that carbon ion beam radioresistant cells were more sensitive to FTD compared with X-ray radioresistant cells. The results of the present study suggested that FTD is involved in carbon ion beam radioresistance, encouraging further study of cellular resistance to charged particle therapy for refractory human cancer.
\end{abstract}

\section{Introduction}

Carbon ion beam radiotherapy is regarded as a cutting-edge technology for the treatment of a number of types of human malignancy, including bone and soft-tissue sarcoma of the head, neck and pelvis, and locally recurrent rectal and

Correspondence to: Dr Kazuhiko Ogawa, Department of Radiation Oncology, Osaka University Graduate School of Medicine, 2-2 Yamadaoka, Suita, Osaka 565-0871, Japan

E-mail: kogawa@radonc.med.osaka-u.ac.jp

Dr Hideshi Ishii, Department of Cancer Profiling Discovery, Osaka University Graduate School of Medicine, Yamadaoka 2-2, Suita, Osaka 565-0871, Japan

E-mail: hishii@gesurg.med.osaka-u.ac.jp

*Contributed equally

Key words: carbon ion beam radiation, trifluorothymidine, rodents, radiosensitizer pancreatic cancer, following surgery (1). Whereas a systemic review indicated that the mechanism by which ionized radiation therapy may induce apoptosis of cancer cells involves redox regulation (2), one study revealed that X-rays induced the production of reactive oxygen species (ROS), and that cancer stem cells and other small fractions of tumor tissues were able to survive following therapy (3). By contrast, carbon ion beam radiotherapy typically exerts antitumor effects directly on cellular organelles, including the nucleus, in which the DNA may be exposed to double-stranded breaks (4). As DNA double-stranded breaks are harmful to cells, this presents a promising therapeutic mechanism via which efficient eradication of tumors is expected (5).

Previous studies have indicated that cancer stem cells serve a function in the therapeutic resistance to $\mathrm{X}$-ray radiation via redox regulation; for example, cancer stem cells express cell surface markers, including cluster of differentiation (CD)13 (6) and CD44 variants (7), and survive by controlling intracellular ROS metabolism following chemotherapy (6,7). CD13 functions as an aminopeptidase $\mathrm{N}$ in the antioxidant pathway, whereas CD44 variants bound to the amino acid transporter $\mathrm{xCT}$ subunit contribute to the maintenance of reduced conditions, which enable cancer cell survival (7). Novel therapeutic approaches targeting redox controls in cancer stem cells have been developed, including the use of sulfasalazine, an inhibitor of xCT-dependent cystine transport (8), and ubenimex, an inhibitor of CD13 (6). In particular, genetic ablation of CD44 or treatment with sulfasalazine was demonstrated to suppress the development of spasmolytic polypeptide-expressing metaplasia and subsequent gastric tumor growth in mouse models of gastric carcinogenesis (8).

Carbon ion beam radiotherapy resistance mechanisms are not fully understood. Although the anticancer mechanism of X-ray radiation (ROS production) facilitates the survival of cancer stem cells, the unique mechanisms involved in the process of cancer cell survival are not mutually exclusive (4). In the present study, an X-ray-resistant cell line (X60) and a carbon ion beam-resistant cell line (C30) were studied using mice. It was revealed that X60 cells were resistant to X-rays and carbon ion beams, whereas $\mathrm{C} 30$ cells were not resistant to 
$\mathrm{X}$-rays, suggesting that X-ray resistance and carbon ion beam resistance exhibit distinct mechanisms. As carbon ion beam radioresistant cells have not been extensively studied to date, the association between carbon ion beam and chemotherapy resistance remains unknown. Evaluation of optimal chemotherapy regimens is important for improving treatment outcomes, as it serves an important function in eliminating metastases and microinvasions, which may cause tumor recurrence (4). For $\mathrm{X}$-ray radioresistance, clinical trials have examined a number of drugs and treatments in order to determine the most effective combination and types; for example, cisplatin (CDDP) and 5-fluorouracil (5-FU) are typically used in combination with $\mathrm{X}$-ray radiotherapy (2-4). A previous study demonstrated that a combination of trifluorothymidine (FTD) and the thymidine phosphorylase inhibitor, tipiracil $\mathrm{HCl}$ (TAS-102), was effective against 5-FU-resistant cancer (9). However, there is not much evidence associated with carbon ion beam radiation, and previous clinical studies have focused on an optimal treatment dose, but not drug selection (1,10-16). In the present study, the effect of FTD, a derivative of deoxythymidine, on carbon ion beam radioresistant cells from rodents was studied. The results of the present study revealed previously uncharacterized features of carbon ion beam radioresistance, suggested the good efficacy of FTD, and encouraged further study of the mechanism of cellular resistance to charged particle therapy and the effects in humans.

\section{Materials and methods}

Cell lines. The NR-S1 mouse squamous cell carcinoma cell line (control) was provided by Dr Koichi Ando (Medicine and Biology Division, Gunma University Heavy Ion Medical Center, Maebashi, Japan). The X60 (X-ray radioresistant) and C30 (carbon ion radioresistant) cells were grown from NR-S1 as described (17) and maintained in Dulbecco's modified Eagle's medium (DMEM; Sigma-Aldrich; Merck KGaA, Darmstadt, Germany) with $10 \%$ fetal bovine serum (FBS; HyClone; GE Healthcare, Logan, UT, USA) and penicillin/streptomycin (Sigma-Aldrich; Merck KGaA), and maintained at $37^{\circ} \mathrm{C}$ in an incubator containing $5 \% \mathrm{CO}_{2}$.

$X$-ray radioresistant cells. X60 cells were established by irradiating NR-S1 cells with 10 Gy X-ray radiation once every 2 weeks. The cells were irradiated with a total dose of $60 \mathrm{~Gy}$ and cultured in DMEM with $10 \% \mathrm{FBS}$ at $37^{\circ} \mathrm{C}$ to $\sim 70 \%$ confluency for 4 weeks following the final irradiation (17).

Carbon ion beam radioresistant cells of rodents. C30 cells were established by irradiating NR-S1 cells with 5 Gy carbon ion beam radiation once every 2 weeks (Fig. 1). The cells were irradiated with a total dose of $30 \mathrm{~Gy}$ and cultured in DMEM with $10 \% \mathrm{FBS}$ at $37^{\circ} \mathrm{C}$ at $\sim 70 \%$ confluency for 4 weeks following the final irradiation.

Cell viability assay with FTD, 5-FU and CDDP. Cell viability assays were performed using Cell Counting Kit-8 (Dojindo Molecular Technologies, Inc., Kumamoto, Japan) according to the manufacturer's protocol. As presented in Fig. 2, cells were plated into 96-well plates at 1,000 cells/well and cultured at $37^{\circ} \mathrm{C}$ overnight. Subsequently, the cells were treated with various concentrations of 5-FU $\left(10,5,2.5,1.25,6.25 \times 10^{-1}\right.$, $3.12 \times 10^{-1}, 1.56 \times 10^{-1}, 7.81 \times 10^{-2}, 3.9 \times 10^{-2}$ and $\left.1.95 \times 10^{-2} \mu \mathrm{M}\right)$, FTD $\left(2 \times 10^{2}, 40,8,1.6,3.2 \times 10^{-1}, 6.4 \times 10^{-2}, 1.28 \times 10^{-2}, 2.56 \times 10^{-3}\right.$, $5.12 \times 10^{-4}$ and $\left.1.02 \times 10^{-4} \mu \mathrm{M}\right)$, and $\operatorname{CDDP}\left(2.5,1.25,6.25 \times 10^{-1}\right.$, $3.12 \times 10^{-1}, 1.56 \times 10^{-1}, 7.81 \times 10^{-2}, 3.9 \times 10^{-2}, 1.95 \times 10^{-2}, 9.76 \times 10^{-3}$ and $4.88 \times 10^{-3} \mathrm{mM}$ ) at $37^{\circ} \mathrm{C}$ for $72 \mathrm{~h}$ with the comparison of parental NR-S1 control. Viable cells were counted using Cell Counting Kit-8 after $2 \mathrm{~h}$ of incubation at $37^{\circ} \mathrm{C}$.

Statistical analysis. Results are expressed as mean \pm standard deviation based on 3 independent experiments. The statistical significance of the results was evaluated using a pairwise t-test with Bonferroni's correction. Analysis was performed using R package software (v3.4.3; date accessed, 30/11/2017; https://www.R-project.org/). $\mathrm{P}<0.05$ was considered to indicate a statistically significant difference.

\section{Results}

Radiation sensitivity of carbon ion beam irradiated radiation-resistant cancer cells. Although it is known that repeated X-ray irradiation results in cancer cells developing $\mathrm{X}$-ray resistance, cancer cells developing resistance following repeated $\mathrm{C}$-ion irradiation has not been demonstrated. In the present study, $\mathrm{C}$-ion-induced radioresistant cancer cells (C30) were established using repetition of $\mathrm{C}$-ion irradiation, and their X-ray and C-ion sensitivity was then evaluated (Fig. 1B). The $\mathrm{C}$-ion resistance of the $\mathrm{C} 30$ cells was significantly increased $(\mathrm{P}<0.01)$, compared with that of the parental NR-S1 cells. The C30 cells were 3.9-fold resistant to $\mathrm{C}$-ion at $5 \mathrm{~Gy}$ compared with the NR-S1 cells. The D10 dose, the radiation dose required to decrease the survival fraction by 0.1 , of $\mathrm{C}$-ion irradiation for the $\mathrm{C} 30$ and NR-S1 cells was 4.9 and $3.9 \mathrm{~Gy}$, respectively. Furthermore, the X-ray sensitivity of the C30 cells was determined (Fig. 1C). Notably, the C30 cells did not acquire significant X-ray resistance compared with the NR-S1 cells. The D10 dose of X-ray for C30 and NR-S1 cells was 7.9 and 7.0 Gy, respectively. The C30 cells were 1.9-fold resistant to X-ray at 10 Gy compared with the NR-S1 cells.

Sensitivity of carbon ion beam radioresistant cells. The present study focused on the difference in radioresistance between X60 and C30 cells, and hypothesized that C30 cells exhibited different radioresistances themselves to that of X60. If X60 and C30 cells exhibited distinct characteristics, the chemosensitivity of the two types of cell may additionally be different. To evaluate the chemosensitivity of X60 and C30 cells, viability assays using CDDP, 5-FU and FTD were performed. Each group was compared with the control NR-S1 group by pairwise t-test with Bonferroni's correction. The analysis revealed that X60 cells were more sensitive to 5-FU compared with the C30 and NR-S1 cells at two points (Fig. 3). C30 cells were significantly more sensitive to FTD compared with the NR-S1 cells (Fig. 4). The cell survival was increased in X60 cells in the concentration of $0.1 \mathrm{mM}$ of CDDP compared with the NR-S1 cells (Fig. 5).

Sensitivity of $X$-ray radioresistant cells. The chemosensitivity of X-ray radioresistant (X60) cells was evaluated. X60 cells were more sensitive to 5-FU, compared with NR-S1 and C30 
A.

30Gy of C-ion

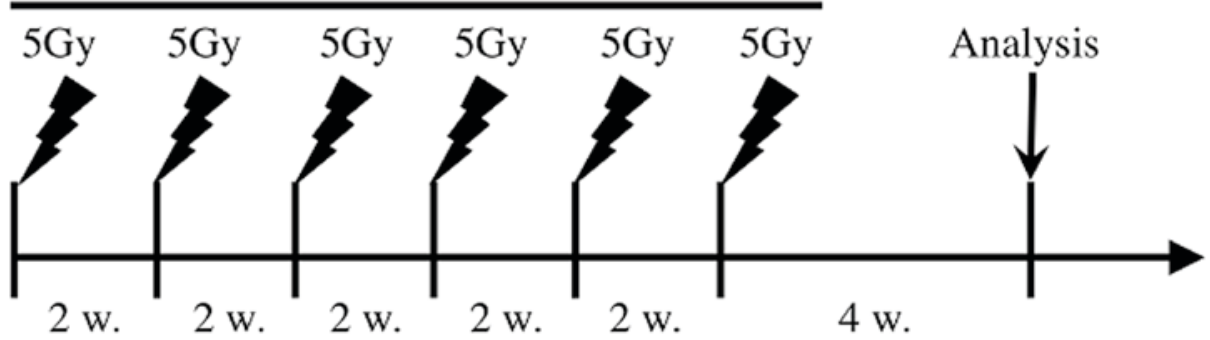

B. Carbon ion beam

C. X-ray

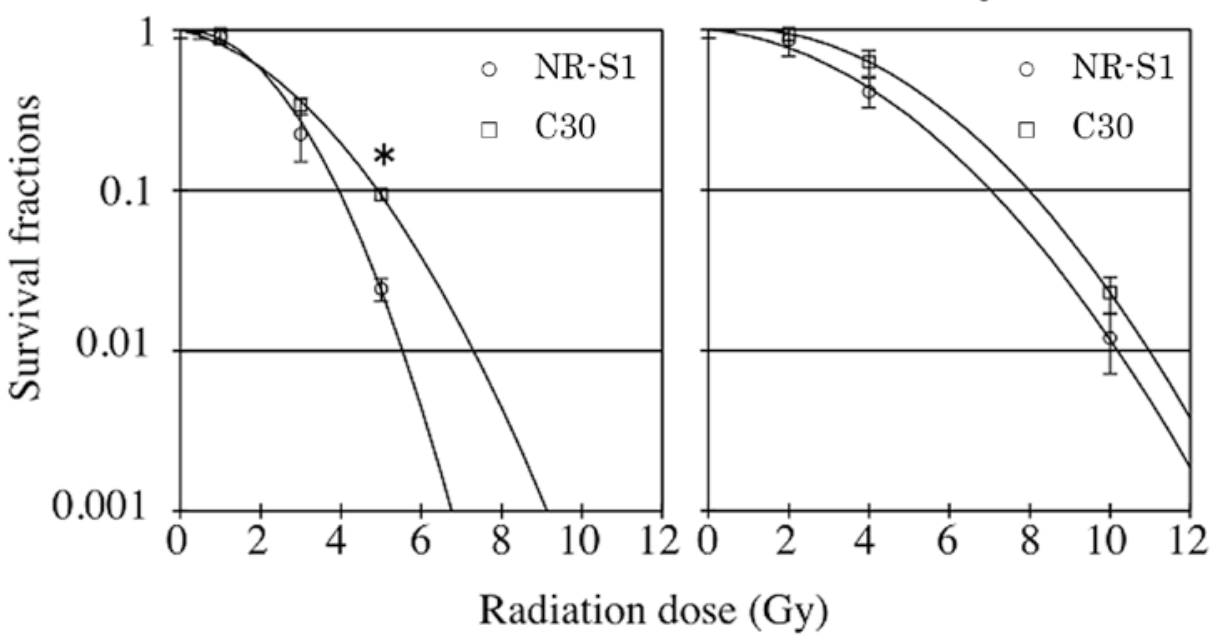

Figure 1. C-ion and X-ray sensitivity of C-ion-resistant cancer cells. (A) Irradiation procedure for establishment of carbon ion-resistant cancer cells. The NR-S1 cells were irradiated using a 30-Gy C-ion beam. At 4 weeks post-irradiation, the irradiated cells were used as the C-ion-resistant cancer C30 cells. (B) C-ion sensitivity of C30 and parental NR-S1 cells. (C) X-ray sensitivity of C30 and NR-S1 cells. The circle and square represent the survival fraction of NR-S1 and $\mathrm{C} 30$ cells, respectively. The statistically significant difference between the cells was detected at 5 Gy $\mathrm{C}$-irradiation. * $\mathrm{P}=1.56 \mathrm{x} 10^{-7}$, $\mathrm{C} 30 \mathrm{vs}$. NR-S1 cells. C-ion, carbon ion.

cells (Fig. 3). By contrast, X60 cells were less sensitive to CDDP and FTD compared with NR-S1 and C30 cells (Figs. 4 and 5). Therefore, X60 and C30 exhibited different chemosensitivity and radiosensitivity characteristics. As previously described (9), 5-FU resistance was not associated with FTD resistance and FTD effectively sensitized C30 cells.

\section{Discussion}

Previous studies have demonstrated that X-ray radiation results in replication stress and arrest, stalling of replication forks and single-strand DNA breaks occur when cell cycle checkpoints are activated $(4,5)$. These biological effects are associated with the production of ROS from cellular organelles such as mitochondria (18). Charged particle therapy, including carbon ion beam radiation, may induce double-strand DNA breaks, and at an increased frequency compared with X-ray radiation, although additive anti-cancer effects are typically observed (4). Carbon ion beam radiotherapy is a useful treatment option for X-ray-resistant cancer (4). For retractable cancer, carbon ion beam radiotherapy does not replace conventional X-ray radiotherapy, as it exhibits an increased risk of damage to normal tissues and has a higher cost (4).
However, the molecular mechanisms involved in this type of therapeutic resistance remain unknown. The current working model of therapeutic resistance [i.e. the mechanism of charged particle therapy-induced double-strand DNA breaks (4)] and its associated genomic effects should be considered, as charged particles exert biological effects on DNA. This difference in carbon ion beam mechanism may affect the cellular characteristics of carbon ion-resistant cells, which as a result may have different chemosensitivity.

In the present study, rodent cell lines that were resistant to carbon ion beam radiation were examined. The results indicated that carbon ion beam radioresistant cells were more sensitive to FTD exposure compared with X-ray radioresistant cells. FTD, a derivative of deoxythymidine, is an antitumor and antiviral agent. As antitumor agents, FTD combined with tipiracil hydrochloride (1:0.5 ratio; TAS-102) are available for use with 5-FU refractory unresectable colorectal cancer (19). Tipiracil may inhibit thymidine phosphorylase in the liver and intestine, resulting in slow FTD degradation and augmentation of FTD bioavailability (19). A previous randomized trial has demonstrated the efficacy of TAS-102 for refractory metastatic colorectal cancer (20). In the present study, FTD was used in an in vitro assay; however, as there are differences between 
Incubate $24 \mathrm{~h} \quad$ Incubate $72 \mathrm{~h}$
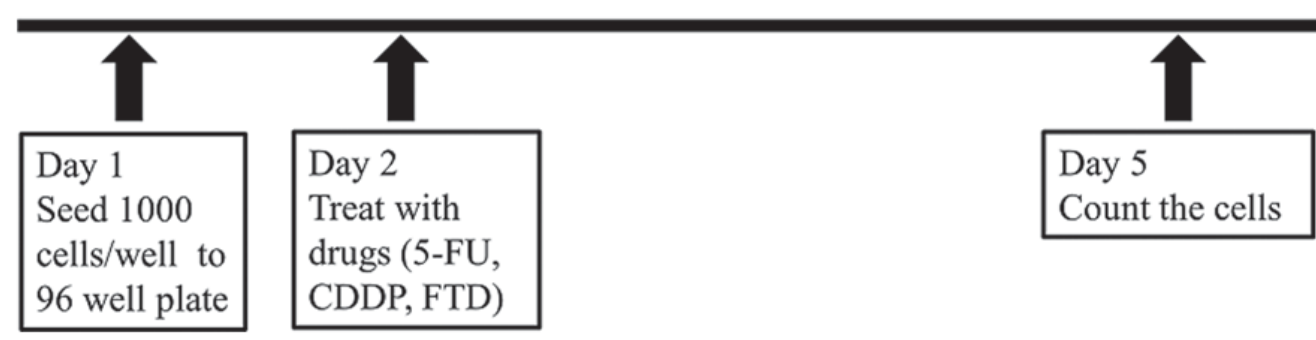

Figure 2. Schema of the protocol. Viability assays were performed with three replicates/assay. NR-S1, X60 and C30 cells were seeded at 1,000 cells/well. After $24 \mathrm{~h}$ of incubation, the specified chemotherapeutic drugs were added in various concentrations $\left(10,5,2.5,1.25,6.25 \times 10^{-1}, 3.12 \times 10^{-1}, 1.56 \times 10^{-1}, 7.81 \times 10^{-2}\right.$, $3.9 \times 10^{-2}$ and $1.95 \times 10^{-2} \mu \mathrm{M}$ of $5-\mathrm{FU} ; 2 \times 10^{2}, 40,8,1.6,3.2 \times 10^{-1}, 6.4 \times 10^{-2}, 1.28 \times 10^{-2}, 2.56 \times 10^{-3}, 5.12 \times 10^{-4}$ and $1.02 \times 10^{-4} \mu \mathrm{M}$ of FTD; $2.5,1.25,6.25 \times 10^{-1}, 3.12 \times 10^{-1}$, $1.56 \times 10^{-1}, 7.81 \times 10^{-2}, 3.9 \times 10^{-2}, 1.95 \times 10^{-2}, 9.76 \times 10^{-3}$ and $4.88 \times 10^{-3} \mathrm{mM}$ of CDDP. Survival was evaluated by counting the number of viable cells using Cell Counting Kit-8 $72 \mathrm{~h}$ after the addition of the drugs. 5-FU, 5-fluorouracil; CDDP, cisplatin; FTD, trifluorothymidine.



Figure 3. Sensitivity to 5-FU. Viability assays of cell lines treated with 5-FU. At least three independent experiments were conducted. ${ }^{\mathrm{P}}<0.05$ for $\mathrm{X} 60 \mathrm{vs.}$ NR-S1 control; ${ }^{* *} \mathrm{P}<0.05$ for C30 vs. NR-S1 control. 5-FU, 5 -fluorouracil.

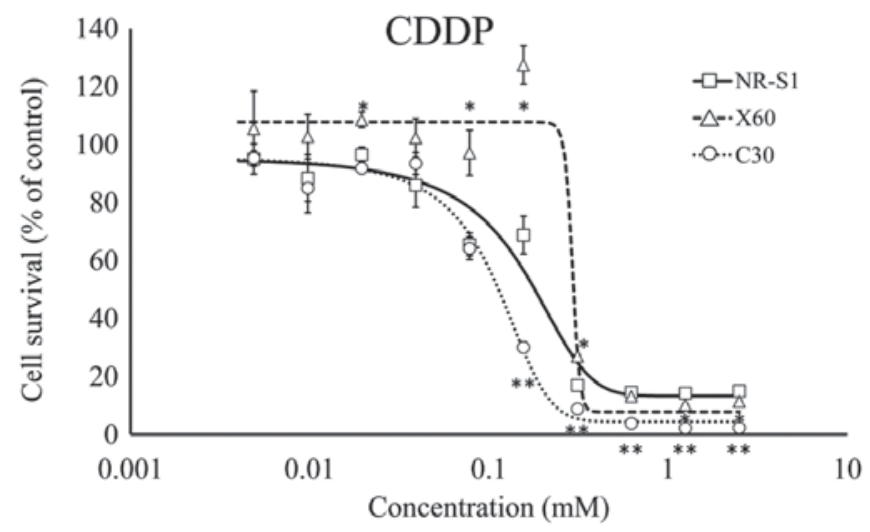

Figure 5. Sensitivity to CDDP. Viability assay of cell lines treated with CDDP. At least three independent experiments were conducted. ${ }^{*} \mathrm{P}<0.05$ for $\mathrm{X} 60$ vs. NR-S1 control; ${ }^{* *} \mathrm{P}<0.05$ for $\mathrm{C} 30$ vs. NR-S1 control. CDDP, cisplatin.

demonstrated to lead to apoptosis and the inhibition of viral replication (19). We hypothesize that carbon ion beam radiation induces random double-stranded DNA breaks in multiple genomic regions; in response, the remaining unaffected coding and non-coding regions, including microRNA, are upregulated to compensate for the damage caused, resulting in resistance to charged particle radiation. However, the potentially active genomic region may incorporate FTD triphosphate, resulting in the induction of apoptosis.

FTD is hypothesized to be effective against 5-FU-resistant cancer (9), and the present study revealed that FTD was effective against carbon ion beam-resistant cancer cells. Treatment with 5-FU was more effective in X-ray-resistant cancer cells than control cells, and 5-FU is typically used with X-ray radiotherapy. FTD may become a good option to use in combination with carbon ion beam radiotherapy instead of 5-FU. Additional in vivo and clinical studies are required to validate these results.

The results of the present study demonstrated that carbon ion beam radioresistant rodent cells are sensitized to FTD exposure. In future, the following studies are required: i) Clinical trials to evaluate charged particle therapy combined with FTD treatment in humans; ii) biomarker identification for the prediction of carbon ion beam radioresistance, including microRNAs in liquid biopsy; and iii) a mechanistic study of carbon ion beam radioresistance. A similar clinical setting phorylated to FTD triphosphate, which has been suggested to integrate into DNA (19). These biological effects have been rodents and humans, clinical trials are required to validate the in vitro results. Furthermore, administration of FTD may effectively sensitize cells to carbon ion beam irradiation although clinical studies in humans are required.

FTD may be converted to FTD monophosphate via thymidine kinase 1 . The FTD monophosphate may be phos- 
may be useful to investigate advanced gastrointestinal cancer following carbon ion beam radiation.

\section{Acknowledgements}

Not applicable.

\section{Funding}

The present study was supported in part by a Grant-in-Aid for Scientific Research from the Ministry of Education, Culture, Sports, Science and Technology (Japan), P-DIRECT (to HI), a Grant-in-Aid from the Ministry of Health, Labor and Welfare (MM), a grant from the National Institute of Biomedical Innovation (MM), and a grant from the Osaka University Drug Discovery Funds (HI). Partial support was received from Taiho Pharmaceutical Co., Ltd. (MM and HI), Evidence Based Medical Research Center through institutional endowments (MM and HI). Part of the study was performed as a research project with heavy ions at NIRS-HIMAC (grant no. 15J183) and supported by the Grants-in-Aid for Scientific Research in Japan Society for the Promotion of Science (grant no. 15K15467).

\section{Availability of data and materials}

The datasets used and/or analyzed during the current study are available from the corresponding author on reasonable request.

\section{Authors' contributions}

The experiments were performed by SJB, KS, JK, KH, KK and MK. The analysis of data was performed by SJB, NN, JK and MK. The manuscript was written by SJB, KS, JK, KH, KK, MK. YD, MM, KO and HI. The study was designed by SJB, YD, MM, KO and HI.

\section{Ethics approval and consent to participate}

Not applicable.

\section{Patient consent for publication}

Not applicable.

\section{Competing interests}

Taiho Pharmaceutical Co., Ltd. and Evidence Based Medical Research Center funding had no role in the study design, data collection or data analysis. However, the company performed a pre-submission review of the manuscript.

\section{References}

1. Kamada T, Tsujii H, Blakely EA, Debus J, De Neve W, Durante M, Jäkel O, Mayer R, Orecchia R, Pötter R, et al: Carbon ion radiotherapy in Japan: An assessment of 20 years of clinical experience. Lancet Oncol 16: e93-e100, 2015.
2. Yasueda A, Urushima $\mathrm{H}$ and Ito $\mathrm{T}$ : Efficacy and interaction of antioxidant supplements as adjuvant therapy in cancer treatment: A systematic review. ntegr. Integr Cancer Ther 15: 17-39, 2016.

3. Skvortsova I, Debbage P, Kumar V and Skvortsov S: Radiation resistance: Cancer stem cells (CSCs) and their enigmatic pro-survival signaling. Semin Cancer Biol 35: 39-44, 2015.

4. Baek SJ, Ishii H, Tamari K, Hayashi K, Nishida N, Konno M, Kawamoto K, Koseki J, Fukusumi T, Hasegawa S, et al: Cancer stem cells: The potential of carbon ion beam radiation and new radiosensitizers (Review). Oncol Rep 34: 2233-2237, 2015.

5. Kunkel TA: DNA replication fidelity. J Biol Chem 279: 1689516898, 2004.

6. Haraguchi N, Ishii H, Mimori K, Tanaka F, Ohkuma M, Kim HM, Akita H, Takiuchi D, Hatano H, Nagano H, et al: CD13 is a therapeutic target in human liver cancer stem cells. J Clin Invest 120: 3326-3339, 2010.

7. Ishimoto T, Nagano O, Yae T, Tamada M, Motohara T, Oshima H, Oshima M, Ikeda T, Asaba R, Yagi H, et al: CD44 variant regulates redox status in cancer cells by stabilizing the $\mathrm{xCT}$ subunit of system xc(-) and thereby promotes Tumor Growth. Cancer Cell 19: 387-400, 2011.

8. Wada T, Ishimoto T, Seishima R, Tsuchihashi K, Yoshikawa M, Oshima H, Oshima M, Masuko T, Wright NA, Furuhashi S, et al: Functional role of $\mathrm{CD} 44 \mathrm{v}-\mathrm{xCT}$ system in the development of spasmolytic polypeptide-expressing metaplasia. Cancer Sci 104: 1323-1329, 2013.

9. Peters CJ: Therapeutic potential of TAS-102 in the treatment of gastrointestinal malignancies. Ther Adv Med Oncol 7: 340-356, 2015.

10. Uhl M, Herfarth K and Debus J: Comparing the use of protons and carbon ions for treatment. Cancer J 20: 433-439, 2014.

11. Cui X, Oonishi K, Tsujii H, Yasuda T, Matsumoto Y, Furusawa Y, Akashi M, Kamada T and Okayasu R: Effects of carbon ion beam on putative colon cancer stem cells and its comparison with X-rays. Cancer Res 71: 3676-3687, 2011.

12. Ishikawa H, Tsuji H, Kamada T, Akakura K, Suzuki H, Shimazaki J and Tsujii H; Working Group for Genitourinary Tumors: Carbon-ion radiation therapy for prostate cancer. Int $\mathrm{J}$ Urol 19: 296-305, 2012.

13. Tsujii $\mathrm{H}$ and Kamada T: A review of update clinical results of carbon ion radiotherapy. Jpn J Clin Oncol 42: 670-685, 2012.

14. Nakano T, Suzuki Y, Ohno T, Kato S, Suzuki M, Morita S, Sato S, Oka K and Tsujii H: Carbon beam therapy overcomes the radiation resistance of uterine cervical cancer originating from hypoxia. Clin Cancer Res 12: 2185-2190, 2006.

15. Mizoe JE, Hasegawa A, Jingu K, Takagi R, Bessyo H, Morikawa T, Tonoki M, Tsuji H, Kamada T, Tsujii H, et al: Results of carbon ion radiotherapy for head and neck cancer. Radiother Oncol 103: 32-37, 2012

16. Jingu K, Tsujii $H$, Mizoe JE, Hasegawa A, Bessho $H$, Takagi R, Morikawa T, Tonogi M, Tsuji H, Kamada T and Yamada S; Organizing Committee for the Working Group for Head-and-Neck Cancer: Carbon ion radiation therapy improves the prognosis of unresectable adult bone and soft-tissue sarcoma of the head and neck. Int J Radiat Oncol Biol Phys 82: 2125-2131, 2012.

17. Sato K, Imai T, Okayasu R and Shimokawa T: Heterochromatin domain number correlates with x-ray and carbon-ion radiation resistance in cancer cells. Radiat Res 182: 408-419, 2014.

18. Chandel NS: Evolution of Mitochondria as Signaling Organelles. Cell Metab 22: 204-206, 2015.

19. Temmink OH, Emura T, de Bruin M, Fukushima M and Peters GJ: Therapeutic potential of the dual-targeted TAS-102 formulation in the treatment of gastrointestinal malignancies. Cancer Sci 98: 779-789, 2007.

20. Mayer RJ, Van Cutsem E, Falcone A, Yoshino T, Garcia-Carbonero R, Mizunuma N, Yamazaki K, Shimada Y, Tabernero J, Komatsu Y, et al: Randomized trial of TAS-102 for refractory metastatic colorectal cancer. N Engl J Med 372: 1909-1919, 2015 